\title{
Relationship Between Degrees Of Erectile Disfunction According To Iief-5 And Color Doppler Ultrasound Image Of Penis Evaluated In The Flaccid Phase
}

\author{
Fahmi N,M (1) Handarini. T,W (2), Pasaribu. U,P(2) Atmadja. H,S(2) Tanodjo T,J ${ }^{(3)}$ \\ ${ }^{1}$ Resident of Study Program of Radiology, Faculty of Medicine, Airlangga University \\ ${ }^{2}$ Staff of Department of Radiology, Faculty of Medicine, Airlangga University \\ ${ }^{3}$ Staff of Department of Andrology, Faculty of Medicine, Airlangga University
}

\begin{abstract}
Background: Erectile dysfunction occurs in $15 \%$ to $50 \%$ of men, and increases with age. International Index of Erectile Function (IIEF-5) questionnaire is a tool for screening and assessing the response to erectile dysfunction treatment. With USG, especially CDUS of the penis, we can evaluate penile vascular condition, by measuring PSV and EDV, we can determine the type of penile vascular disturbance whether it comes from arteries or veins or combination of both. Objective: This study aims to determine relationship between degree of erectile dysfunction according to IIEF-5 and color doppler ultrasound image of the penis evaluated in the flaccid phase. Methods : This study is a prospective observational study conducted on 30 samples with varying degrees of erectile dysfunction. USG examination was performed in flaccid phase with peak systolic velocity (PSV), cavernous artery diameter, end-diastolic velocity (EDV), and resistive index (RI) parameters. Data normality test done by a regression test with dummy variables. Results : We found 30 patients with various degrees of erectile dysfunction, mild (36\%), mild-moderate $(27 \%)$, moderate $(27 \%)$, severe $(10 \%)$. There's strong relationship between degree of erectile dysfunction and PSV value (72.32\%). The average RI value of study was 0.77 . There's no correlation between degree of erectile dysfunction and cavernous artery diameter. Conclusion : There's a strong relationship between degree of erectile dysfunction with PSV and RI value. There's no significant relationship between degree of erectile dysfunction and the cavernous artery diameter.
\end{abstract}

Published by IJRP.ORG. Selection and/or peer-review under responsibility of International Journal of Research Publications (IJRP.ORG)

Keywords : Penile Color Doppler Ultrasound, Erectile Dysfunction, Flaccid Phase, IIEF-5

\section{Background}

Ultrasound is an examination technique based on sound waves. This method is relatively safer because it does not have radiation effects and is more flexible due to the relatively small size of the instrument. A penile ultrasound is a high-performance, non-invasive imaging modality. In addition, functional changes in penile blood flow as seen in erectile dysfunction can be studied precisely by the color doppler ultrasound method. ${ }^{1}$

Erectile dysfunction occurs in $15 \%$ to $50 \%$ of men, and increases with age. Erectile dysfunction is caused by many organic factors, especially in the vascular system. The elasticity of the blood vessels of the penis plays an important role in an erection as it enlarges and fills with blood. ${ }^{1}$ The International Index of Erectile Function (IIEF-5) is a tool for screening and assessing treatment response., ${ }^{2,3}$ The IIEF-5 questionnaire consists of the 5 psychometrically valid and reliable questions regarding erectile function, orgasm, sexual drive, sexual satisfaction, and general satisfaction.

Vascular disorders that are evaluated using ultrasound can be distinguished based on the findings obtained, namely due to arterial insufficiency, venous leakage, or a combination of the two. can be in the form of genital self-stimulation, audiovisual, oral medication (PDE-5i), or in the form of intracavernous injection which will then be carried out further examination using CDUS. According to several studies, CDUS examination can describe the condition of the penis such as when it is erect. By using ultrasound, especially CDUS on the penis, it can be evaluated regarding the penile vascular condition, by measuring the PSV and EDV it will be able to determine the type of disturbance in the penile vascular whether it is from disturbances in the arteries, veins or a combination of both..$^{9,10}$ Based on the facts, the authors are interested to determine the relationship between the 
degree of erectile dysfunction according to IIEF-5 and the color doppler ultrasound image of the penis evaluated in the flaccid phase.

\section{Method}

The study was conducted at Dr. Soetomo Hospital Surabaya in December 2019 - March 2020. This is a prospective observational study. The number of study samples were all erectile dysfunction patients who were consulted to the Ultrasound Radiology Division of the Dr. Soetomo Hospital for a penis ultrasound examination in December 2019 - March 2020, which met the inclusion criteria. With inclusion criteria: men who have mildsevere erectile dysfunction, aged 30-65 years, married and still living with their wives, are still sexually active, willing and signing an informed consent. Patients who had a psychological disorder, spinal cord injury, had a history of life-threatening heart attacks, strokes, or arrhythmias, or had penile anatomic abnormalities that made examination difficult were excluded from this study. Vascular disorders that cause erectile dysfunction can be evaluated using a supporting examination in the form of ultrasonography (USG). On the ultrasound examination, there will be two conditions, namely when the condition is flaccid and erect. The erectile phase of the patient will be stimulated by the use of intracavernous injection drugs, as stimulation is known to have an effective response to evaluate the vascular condition of the erectile tissue. A vascular evaluation will be carried out 5 - 10 minutes after injection for optimal results. After the examination is carried out, the patient will be monitored and evaluated until a condition of detumescence occurs in the patient..$^{6,7,11}$, in this study the evaluation was only carried out in the flaccid phase, according to the results of several studies which stated that evaluation of the flaccid vase alone was considered to be able to describe the condition of the penis just like when it is in an erect condition. ${ }^{9,10}$ Ultrasonography (USG) is performed in the flaccid phase. The results of the ultrasonography examination will obtain several parameter results, including peak systolic velocity (PSV), artery diameter, end-diastolic velocity (EDV), and resistive index (RI) and will be documented. The research was conducted in the USG Division of the Integrated Diagnostic Center Building, Dr. Soetomo Surabaya. The data obtained will be inputted and processed with the SPSS 20.0 for Windows ${ }^{\text {TM }}$ program. Descriptive data presentation is carried out to determine the characteristics of all data held to determine the mean and standard deviation. The data normality test was performed on penile vascularization data (PSV, arterial diameter, EDV, and RI) with the regression test with dummy variables. Normal distribution if $p>0.05$. Meanwhile, the data homogeneity test was analyzed using the Levene test. The data variant is homogeneous if $\mathrm{p}>0.05$.

3. Result

In this study, there were 30 patients with mild to severe erectile dysfunction, 11 patients with mild erectile dysfunction, 8 patients each with mild to moderate erectile dysfunction and 3 patients with severe erectile dysfunction. The youngest patient was 40 years old and the oldest was 61 years. Measurement of PSV, EDV, RI and cavernous artery diameter were carried out at the USG Division of Radiology Installation at dr. Sutomo Hospital Surabaya uses a General Electric Type E9 ultrasound machine, the examination was carried out from December 2019 to March 2020. The results of the study are as follows. The mean age was 49.01 years, where the youngest age for the sample was 40 years old, while the oldest age was 62 years.

Tabel 5.1 Sample distribution based on the age

Age range $\quad$ Number of sample

\begin{tabular}{lc}
\hline $40-45$ & 8 \\
$46-50$ & 8 \\
$51-55$ & 13 \\
$56-61$ & 1 \\
\hline
\end{tabular}

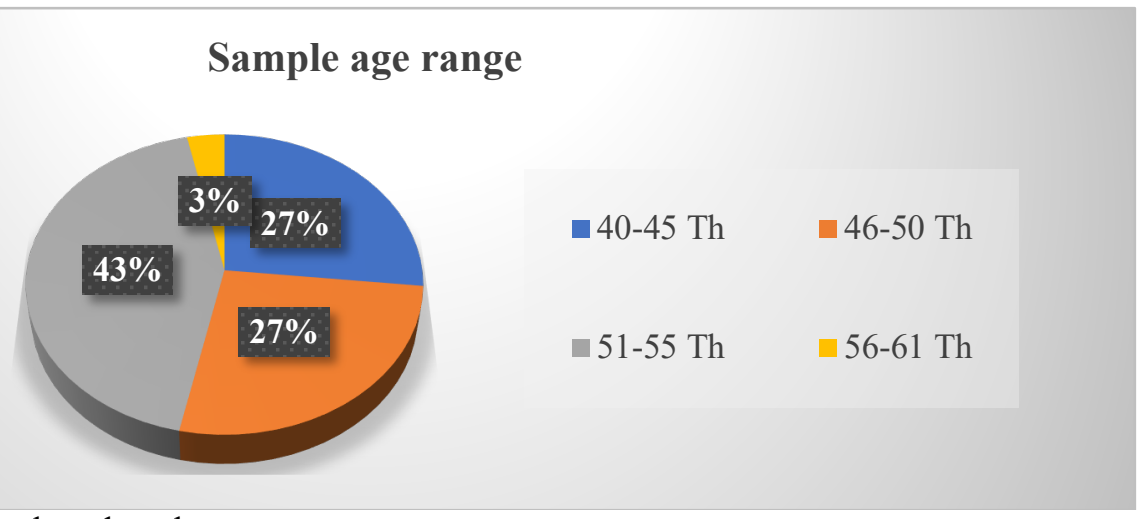

Figure 5.1. Sample distribution based on the age 
From a total of 30 research samples obtained, there were 11 people with mild erectile dysfunction, 8 people with mild to moderate erectile dysfunction, 8 people with moderate erectile dysfunction, and the remaining 3 people had severe degrees of erectile dysfunction.

Table 5.2 Sample distribution based on the degree of erectile dysfunction

\begin{tabular}{cc}
\hline Degree of erectile dysfunction (IIEF-5) & Number of sample \\
\hline MILD & 11 \\
MILD - MODERATE & 8 \\
MODERATE & 8 \\
SEVERE & 3 \\
\hline
\end{tabular}

Sample distribution based on the degree of erectile dysfunction

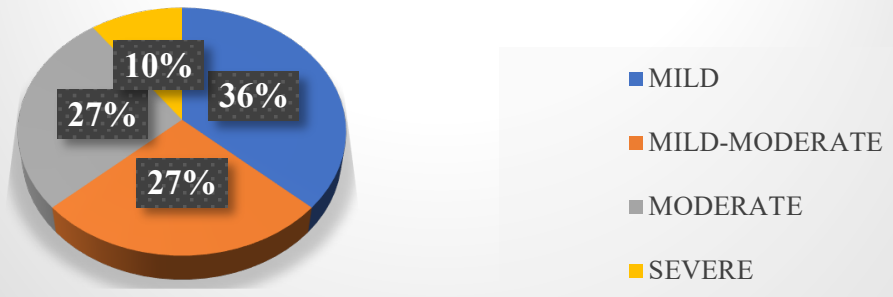

Figure 5.2. Sample distribution based on the degree of erectile dysfunction

From a total of 11 patients with mild erectile dysfunction who underwent Doppler ultrasound examination, the PSV results were obtained with a mean value of $14.3 \mathrm{~cm} / \mathrm{s}$. At the grade of mild-moderate erectile dysfunction in 8 patients, the mean PSV value at this degree was $12.4 \mathrm{~cm} / \mathrm{s} .8$ patients with moderate erectile dysfunction obtained a mean PSV value of $9.1 \mathrm{~cm} / \mathrm{s}$. Meanwhile, for 3 patients, the average PSV value was 7.2 $\mathrm{cm} / \mathrm{s}$.

Table 5.3 Peak Systolic Velocity Based on the Degree of Erectile Dysfunction

Degree of erectile dysfunction (IIEF-5)

\section{MEAN}

\begin{tabular}{cc}
\hline MILD & $14,3 \pm 3,6$ \\
MILD-MODERATE & $12,4 \pm 1,6$ \\
MODERATE & $9,1 \pm 1,9$ \\
SEVERE & $7,2 \pm 2,0$ \\
\hline
\end{tabular}

Peak Systolic Velocity Based on the Degree of Erectile Dysfunction

20

0

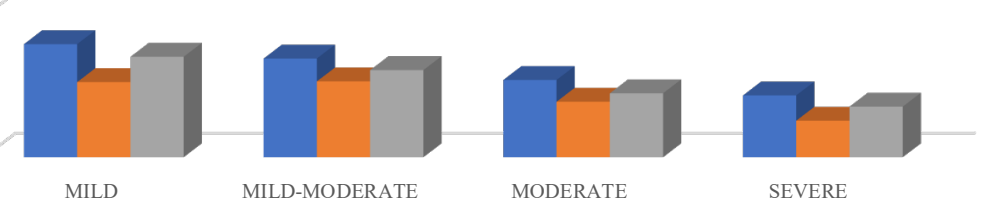

$\mathrm{CM} / \mathrm{S}$

Figure 5.3. Peak Systolic Velocity Based on the Degree of Erectile Dysfunction 
The following is the End Diastolic Velocity value based on the degree of erectile dysfunction in this study.

Table 5.4 End Diastolic Velocity Based on the Degree of Erectile Dysfunction

Degree of erectile dysfunction (IIEF-5)

$$
\text { MILD }
$$

MILD-MODERATE

MODERATE

SEVERE

\section{MEAN}

$2,1 \pm 1,2$

$2,9 \pm 1,1$

$1,4 \pm 1,5$

$1,9 \pm 1,4$

End Diastolic Velocity Based on the Degree of Erectile Dysfunction

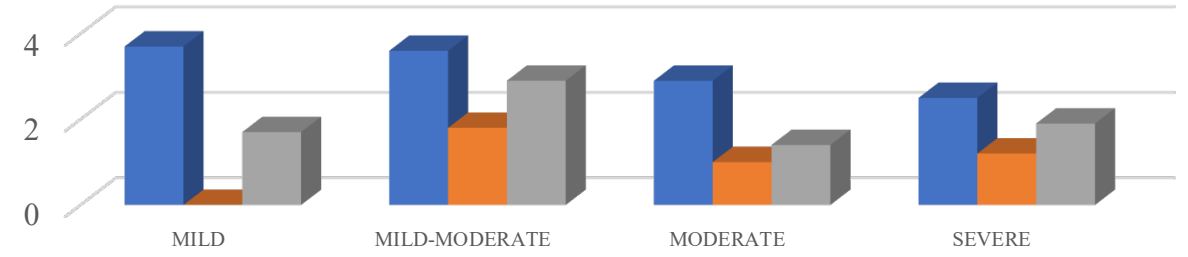

$\mathrm{CM} / \mathrm{S}$

-HIGHEST $\square$ LOWEST

Figure 5.4 End Diastolic Velocity Based on the Degree of Erectile Dysfunction

From a total of 11 patients with mild erectile dysfunction who underwent a Doppler ultrasound examination, the mean RI value was 0.86 , for mild to moderate degrees the mean of RI value was 0.79 .8 patients with moderate degrees of erectile dysfunction obtained the mean of RI value of 0.73 , and the mean of RI value in the severe degrees was 0.73 .

Table 5.5 RI Value Based on the Degree of Erectile Dysfunction

\section{Degree of erectile dysfunction (IIEF-5) MEAN}

$\begin{array}{ll}\text { MILD } & 0,83 \pm 0,07\end{array}$

$\begin{array}{cc}\text { MILD-MODERATE } & 0,79 \pm 0,13 \\ \text { MODERATE } & 0,73 \pm 0,19 \\ \text { SEVERE } & 0,73 \pm 0,03\end{array}$

RI Value Based on the Degree of Erectile Dysfunction

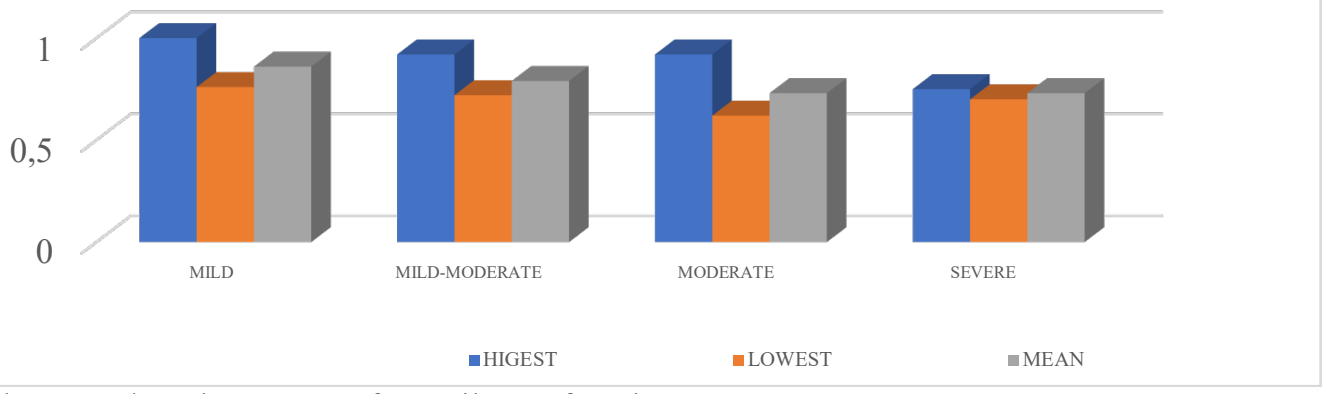

Figure 5.5. RI Value Based on the Degree of Erectile Dysfunction 
In the mild degree group of 11 patients, the largest diameter was $0.11 \mathrm{~cm}$, the smallest was 0.08 with the mean of diameter was $0.093 \mathrm{~cm}$. In the mild-moderate degrees the largest diameter was $0.11 \mathrm{~cm}$, the smallest was $0.07 \mathrm{~cm}$ and the mean was $0.074 \mathrm{~cm}$, while at moderate degrees the largest diameter was $0.10 \mathrm{~cm}$, the smallest was $0.07 \mathrm{~cm}$ with the mean diameter was $0.083 \mathrm{~cm}$. In the severe degree the largest diameter was $0.08 \mathrm{~cm}$ and the smallest was $0.07 \mathrm{~cm}$ with the mean was $0.073 \mathrm{~cm}$.

Table 5.6 Diameter of Cavernous Artery Based on the Degree of Erectile Dysfunction

Degree of erectile dysfunction (IIEF-5)

MILD

MILD-MODERATE

MODERATE

SEVERE

\section{MEAN}

$$
\begin{array}{r}
0,093 \pm 0,017 \\
0,074 \pm 0,03 \\
0,083 \pm 0,017 \\
0,073 \pm 0,08
\end{array}
$$

Diameter of Cavernous Artery Based on the Degree of Erectile Dysfunction

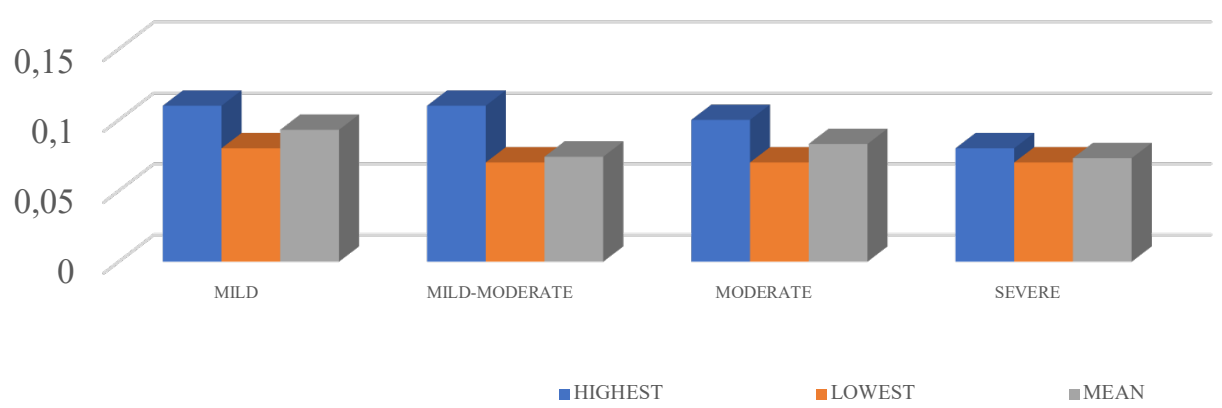

Figure 5.6. Diameter of Cavernous Artery Based on the Degree of Erectile Dysfunction

Results from PSV, EDV, RI, cavernous artery diameter, are ratio data. The normality test will be carried out on all types of ratio data. The normality test used the Breusch-Pagan Test because the data tested were 30 samples.

Table 5.7. The result of normality test on PSV, EDV, RI, and the diameter of cavernous artery

\begin{tabular}{ccc}
\hline No & Variables & P-V value \\
\hline 1 & PSV & 0,55 \\
2 & EDV & 0,32 \\
3 & RI & 0,30 \\
4 & Diameter of cavernous artery & 0,40 \\
\hline
\end{tabular}

From table 5.7 above, it can be concluded that the distribution of data from PSV, EDV, RI and cavernous artery diameter is normal, because the p-value of all the data above is greater than 0.05 .

By using the regression statistical test with dummy variables to determine the relationship between the degree of erectile dysfunction and the Peak Systolic Velocity, it can be concluded that the heavier the degree of erectile dysfunction, the smaller mean of PSV value, which means that the degree of erectile dysfunction has a $72.32 \%$ relationship with the PSV value of patients, with a 95\% confidence interval. By using the regression statistical test with a dummy variable to determine the relationship between the degree of erectile dysfunction and the Restrictive Index, the p-value is 0.03075 with an adjusted R-square of $20.28 \%$. This means that with a confidence interval of $95 \%$, the regression test results are significant that the degree of DE affects the mean of RI by $20.28 \%$ and the rest $(79.62 \%)$ of the mean RI is influenced by other things that are not in the model. By using the regression statistical test with the dummy variable to determine the relationship between the degree of erectile dysfunction and the diameter of the cavernous artery, the p-value is 0.1539 with an adjusted R-square of $8.54 \%$. This means that with a confidence level of $95 \%$, the regression test results are significant that the degree of ED has no relationship with the diameter of the cavernous arteries. 


\section{Discussion}

In this study, there were 30 patients with erectile dysfunction with various degrees, 11 patients $(36 \%)$ mild degree, 8 patients $(27 \%)$ moderate, and 3 severe $(10 \%)$ patients. In terms of age from a total of 30 patients, the largest age range is 51-55 years with a percentage of $43 \%$, then the age range is 40-45 and 46-50 with a percentage of $27 \%$, this is in accordance with various previous studies, including study from Aversa, A et. al, Gatkin, M et a and Vardi, Y which stated that the incidence of erectile dysfunction in various countries increases with age. ${ }^{10,12,13}$

In this study, there was a strong relationship between the degree of erectile dysfunction and its Peak Systolic Velocity value $(72.32 \%$ ) with a $95 \%$ confidence interval, this is in accordance with previous study from Li-Da Chen et al which stated that the more severe degree of a person's erectile dysfunction, the PSV value will decrease further, in this study it was also obtained that the PSV cut off value for patients with severe erectile dysfunction was $8.2-8.9 \mathrm{~cm} / \mathrm{s}$, this is in accordance with the findings in this study where patients with severe erectile dysfunction were obtained the mean PSV value was $7.2 \mathrm{~cm} / \mathrm{s}$.

PSV shows the speed of blood flow in an artery in the systole phase, where in this case the volume of blood flowing in the cavernous artery plays an important role in the process of an erection where the blood flow will fill and expand the corpora cavernosa in the erection process. Low blood flow (velocity) in the cavernous arteries is closely related to the disruption of the erectile process in a person which can ultimately lead to erectile dysfunction. ${ }^{14}$ In this study, a moderate relationship between the degree of erectile dysfunction and the Restrictive Index value was obtained, namely $20.28 \%$. Where the mean RI value obtained in this study was 0.77 , this is also in accordance with the results of study in 2017 where the RI value will decrease according to the degree of erectile dysfunction suffered by the patient.

In this study, the mean age value of End Diastolic Velocity at mild degrees was $2.1+/-1.2 \mathrm{~cm} / \mathrm{s}$, moderate lightness was $2.9+/-1.1 \mathrm{~cm} / \mathrm{s}$, moderate $1.4+/-1,5 \mathrm{~cm} / \mathrm{s}$ and a weight of $1.9+/-1.4 \mathrm{~cm} / \mathrm{s}$. The weak relationship between the degree of erectile dysfunction and EDV in this study can be influenced by several factors, including the technical examination that only evaluates the penis in the flaccid phase, while many other studies have evaluated the penis in the flaccid phase and erection with stimulation using intra-cavernous prostaglandin injection (ICI). In theory, End Diastolic Velocity is the velocity of blood flow in the diastolic phase. Together with the peak systolic velocity, these two things affect the value of the restrictive index (RI) with the formula RI $=(P S V$ EDV)/PSV. In contrast to the very sensitive PSV identifying patients with arterial insufficiency, EDV is more influenced by the resistance of the vein of a blood vessel.

Theoretically, Erectile Dysfunction can be divided into three causes, namely due to disorders of the arteries, disorders of the veins/venous leakage and a combination of both. In erectile dysfunction patients with causes of arterial disorders, there was no significant change in EDV or EDV values were still within normal limits, conversely, in patients with erectile dysfunction due to venous leakage, a higher EDV value would be obtained because of the resistance that occurred in the veins. It is not maximal resulting in high venous flow, whereas erectile dysfunction with mixed causes cannot be assessed using ultrasound because venous competence cannot be evaluated in patients who have experienced arterial insufficiency. (Jung, D.C., et all. 2018)

In this study, there was no relationship between the degree of erectile dysfunction and the diameter of the cavernous arteries, this is in accordance with the results of a study by SI Wahl, MB Rubin, CW Bakal in 1997 which stated that the mean diameter of the cavernous artery in patients with erectile dysfunction in the United States is $0.3-0.7 \mathrm{~mm}$, which is relatively equal to the diameter of the cavernous arteries in healthy people. Data on the average value of cavernous artery diameter in Asians have not been obtained, in 1995 Kadioğlu A, Erdoğru $\mathrm{T}$, Karşidağ K conducted a study on the increase in PSV and cavernous artery diameter in 23 patients with erectile dysfunction and DM in the flaccid and erectile phases compared to normal people, with the result of increased PSV and cavernous artery diameter of the flaccid phase compared to the erectile phase of ED patients with DM lower than normal patients. In this study, we only carried out the examination in the flaccid phase, it was not possible to evaluate the increase in the diameter of the cavernous arteries compared to the erection phase. ${ }^{15}$

\section{Conclusion}

Based on statistical analysis using a regression test with a dummy variable with a confidence interval of $95 \%$, it can be concluded that there is a strong relationship $(72.32 \%)$ between the degree of erectile dysfunction and peak systolic velocity. There is a moderate relationship $(20.28 \%$.) between the degree of erectile dysfunction and the Restrictive Index. There was no significant relationship between the degree of erectile dysfunction and the diameter of the cavernous arteries. The value of End Diastolic Velocity can still be measured in the flaccid phase, although the numbers obtained are relatively low or close to 0 . The author suggests that further research is necessary, especially to evaluate the penile doppler image of patients with erectile dysfunction in the erectile phase with a more homogeneous sample distribution.

\section{Acknowledgment}

I would like to dedicate my gratitude to all the lecturers of Radiology, Faculty of Medicine Universitas Airlangga, my family, and my friends for their endless support.

7. Conflicts of interest

The author declares that there is no conflicts of interest

\section{Reference}

1. A.L Baert, leuven et al,. 2008. Color doppler ultrasound of the penis. New York: Springer Berlin Heidelberg 
2. Rosen, R.C., Cappelleri, J.C., Smith, M.D., Lipsky, J. and Peña, B.M. 1999. "Development and evaluation of an abridged, 5-item version of the international index of erectile function (iief-5) as a diagnostic tool for erectile dysfunction." International Journal of Impotence Research. 19-326.

3. Hafez, E.S.E. and Hafez, S.D. 2005. "Erectile dysfunction: anatomical parameters, etiology, diagnosis, and therapy." Archives of Andrology. 15-31.

4. Pastuszak, A.W. 2014. "Current diagnosis and management of erectile dysfunction." Current Sexual Health Reports. 164-176.

5. Yafi, F.A., Jenkins, L., Albersen, M., Corona, G., Isidori, A.M., Goldfarb, S., Maggi, M., Nelson, C.J., Parish, S., Salonia, A., Tan, R., Mulhall, J.P. and Hellstrom, W.J.G. 2016. "Erectile dysfunction'. ." Nature Reviews Disease Primers.

6. Mutnuru, P.C., Ramanjaneyulu, H.K., Susarla, R., Yarlagadda, J., Devraj, R. and Palanisamy, P. 2017. "Pharmaco penile duplex ultrasonography in the evaluation of erectile dysfunction." Journal of clinical and diagnostic research: TC07-TC10.

7. Jung, D.C., Park, S.Y. and Lee, J.Y. 2018. "'Penile doppler ultrasonography revisited'." Ultrasonography 1624.

8. Yamaçake, K.G.R., Carneiro, F., Cury, J., Lourenço, R., Françolin, P.C., Piovesan, A.C., Srougi, M., Nahas, W.C. and Antonopoulos, I.M. 2018. "'Low-intensity shockwave therapy for erectile dysfunction in kidney transplant recipients. A prospective, randomized, double blinded, sham-controlled study with evaluation by penile Doppler ultrasonography." international Journal of Impotence Research.

9. Sen, J., Singh, R., Airon, R.K. and Godara, R. 2007. "Colour doppler sonography of flaccid penis in evaluation of erectile dysfunction." Asian Journal of Surgery. 122-125.

10. Gatkin, M. and Sopotov, A. 2017. "Doppler test in penis flaccid state to asses erectile dysfunction severity and shockwave treatment outcomes'. ." The Journal of Sexual Medicine S331-S332.

11. Kalyvianakis, D. and Hatzichristou, D. 2017. "Low-intensity shockwave therapy improves hemodynamic parameters in patients with vasculogenic erectile dysfunction: a triplex ultrasonography-based sham-controlled trial'. ." The Journal of Sexual Medicine 891-897.

12. Aversa, A., Bruzziches, R., Francomano, D., Natali, M., Gareri, P. and Spera, G. 2010. "Endothelial dysfunction and erectile dysfunction in the aging man." International Journal of Urology: Official Journal of the Japanese Urological Association 38-47.

13. Vardi, Y., Dayan, L., Apple, B., Gruenwald, I., Ofer, Y. and Jacob, G. 2009. "Penile and systemic endothelial function in men with and without erectile dysfunction. ." European Urology. 979-985.

14. Kim Sae Chul, 1999, Recent advancement in diagnosis of vasculogenic impotance, Departemen of urologi Chung-ang University seul, Korea

15. Kadioğlu A 1 , Erdoğru T, Karșidağ K, 1995, “ Evaluation of penile arterial system with color Doppler ultrasonography in nondiabetic and diabetic males" acces from:https://www.ncbi.nlm.nih.gov/pubmed/7656908 